\title{
Influence of miscut direction on magnetic anisotropy of magnetite films grown on vicinal MgO (100)
}

\author{
V. O. Golub, ${ }^{1}$ V. V. Dzyublyuk, ${ }^{1}$ A. I. Tovstolytkin, ${ }^{1}$ S. K. Arora, ${ }^{2, a)}$ R. Ramos, ${ }^{2}$ \\ R. G. S. Sofin, ${ }^{2}$ and I. V. Shvets ${ }^{2}$ \\ ${ }^{1}$ Institute of Magnetism, $36 \mathrm{~b}$ Vernadsky Blvd., Kyiv 03142, Ukraine \\ ${ }^{2}$ CRANN, School of Physics, Trinity College Dublin, Dublin 2, Ireland
}

(Presented 21 January 2010; received 9 November 2009; accepted 28 December 2009; published online 30 April 2010)

\begin{abstract}
Ferromagnetic resonance (FMR) was used to study the influence of miscut direction on the in-plane fourfold and uniaxial magnetic anisotropies of epitaxial $\mathrm{Fe}_{3} \mathrm{O}_{4}$ films grown on vicinal $\mathrm{MgO}(100)$ surfaces. In-plane angular dependent measurements at room temperature on $\mathrm{Fe}_{3} \mathrm{O}_{4}$ films on two different miscut directions (miscut along $\langle 011\rangle$ and $\langle 010\rangle$ directions) of $\mathrm{MgO}$ showed the presence of both in-plane fourfold (cubic) and in-plane uniaxial anisotropies. Temperature dependent FMR investigations show that the in-plane fourfold anisotropy constant $\left(K_{4}\right)$ is approximately the same for both samples at room temperature. The magnitude of $K_{4}$ increases gradually with decreasing temperature and changes sign from negative to positive values at $130 \mathrm{~K}$. This behavior is consistent with that of bulk magnetite. In addition to $K_{4}$, we find an additional uniaxial component $\left(K_{2}\right)$, whose strength is quite different for the different miscut directions. The magnitude of $K_{2}$ shows very little or no temperature dependence. The observed differences in the magnitude of $K_{2}$ with miscut directions are related to the changes in the antiphase boundary structure and altered magnetic configurations at the boundaries. @ 2010 American Institute of Physics. [doi:10.1063/1.3355890]
\end{abstract}

\section{INTRODUCTION}

Vicinal surfaces offer an interesting opportunity for use as templates for the growth of magnetic nanostructures and manipulating them by altering the periodicity of the stepedge array. ${ }^{1,2}$ Periodicity of the step-edge arrays can be effectively controlled through the magnitude and direction of miscut angle and subsequent heat treatment. There have been several reports on the investigations of magnetic properties of epitaxial magnetic thin films on vicinal surfaces owing to their importance in technological applications and also from a fundamental viewpoint. ${ }^{3-8}$ The magnetic anisotropy of these films is affected by the presence of a regular step-edge array. Previous investigations of magnetic anisotropy of magnetic thin films on vicinal substrates suggest that the presence of a parallel step-edge array induces a uniaxial anisotropy. Magnetization associated with this anisotropy is either parallel $\mathrm{l}^{3,7}$ or perpendicular ${ }^{4,8}$ to the step-edge direction.

Magnetite $\left(\mathrm{Fe}_{3} \mathrm{O}_{4}\right)$, being a half metallic ferromagnet with a high Curie temperature $(858 \mathrm{~K})$, is an important material for spin-electronic applications. ${ }^{9}$ There have been several reports on the study of magnetic properties of the epitaxial magnetite films which are strongly affected by the presence of antiphase boundaries (APBs) and are also dependent on growth method. ${ }^{10-13}$ In a previous investigation, we have shown that the magnetic properties of magnetite films grown on vicinal $\mathrm{MgO}$ (100) substrates are dependent on the strength of miscut angle and the in-plane easy axis of magnetization is perpendicular to the step edges. ${ }^{14}$ The in-plane uniaxial anisotropy was found to be inversely proportional to the thickness of the films. In this report, we provide a com-

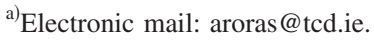

parative study of the step edges induced in-plane uniaxial anisotropy of $\mathrm{Fe}_{3} \mathrm{O}_{4}$ films on the direction of miscut and temperature.

\section{EXPERIMENTAL}

The $\mathrm{Fe}_{3} \mathrm{O}_{4}$ films of $45 \mathrm{~nm}$ thickness were deposited on single crystal $\mathrm{MgO}$ (100) substrates using an oxygen plasma assisted molecular beam epitaxy technique. The $\mathrm{MgO}$ substrates used in the present study had a miscut of $2.86^{\circ}$ and $2^{\circ}$ along the $\langle 010\rangle$ and $\langle 011\rangle$ crystallographic directions, respectively. Details of the epitaxial growth of $\mathrm{Fe}_{3} \mathrm{O}_{4}$ films and the heat treatment to obtain a regular step-edge array on $\mathrm{MgO}$ substrates are given elsewhere. ${ }^{11,15}$ Films were found to be highly stoichiometric as confirmed from in situ reflection high energy electron diffraction (RHEED) and ex situ high resolution x-ray diffraction measurements. Ferromagnetic resonance (FMR) measurements were performed at 9.44 $\mathrm{GHz}$ on $3 \times 3 \mathrm{~mm}^{2}$ size samples using an ELEXSYS -E500 electron paramagnetic resonance (EPR) spectrometer equipped with a goniometer and a cryostat to enable angular dependent as well as temperature dependent FMR measurements to be carried out. ${ }^{16}$

For both samples studied, we use the following unified coordinate system, which is shown in Fig. 1. The $x$-axis coincides with the in-plane uniaxial easy axis (which is perpendicular to the step edge), $\alpha$ is the angle between $x$-axes and [010] crystallographic direction, and $\varphi$ is the angle between the $x$-axis and the projection of magnetization on the film plane.

To study the in-plane anisotropy, we measured the dependence of the resonance field on the direction of the external magnetic field $\boldsymbol{H}$ as it was rotated within the plane of the 


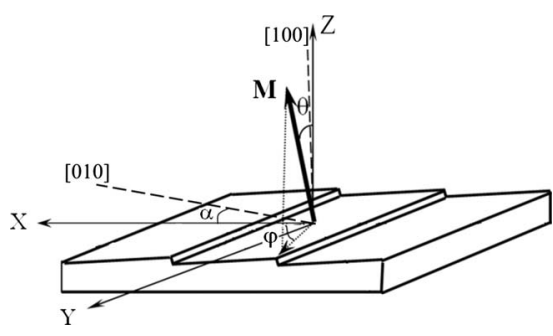

FIG. 1. Schematic diagram of a vicinal MgO (100) substrate and orientation of various axes used in the FMR experiment and data analysis.

film $\left(\theta=90^{\circ}, \varphi=\right.$ var) (Fig. 1). In addition, the temperature dependence of the FMR spectra was studied for the case where $\theta=0^{\circ}$.

\section{RESULTS AND DISCUSSIONS}

Well-characterized $45 \mathrm{~nm}$ thick $\mathrm{Fe}_{3} \mathrm{O}_{4}$ films on single crystal $\mathrm{MgO}$ (100) substrates with miscut directions along $\langle 011\rangle$ and $\langle 010\rangle$ crystallographic directions (referred hereafter as sample 1 and sample 2) were subjected to in-plane and out of plane FMR measurements in the temperature range of 90-300 K. A single FMR line was observed for both samples investigated at each temperature. The in-plane angular dependence of the resonance field $H_{\text {res }}$ measured at different temperatures is shown in Fig. 2 for both samples. One can clearly see that the fourfold cubic anisotropy as expected for a cubic crystal is present. As noted from Fig. 2(a) for sample 1 , the resonance field $H_{\text {res }}$ for [0-11] direction $\left(90^{\circ}\right.$ with respect to miscut direction) is nonequivalent to that of [011] crystallographic direction. This shows that in addition to the cubic anisotropy, an in-plane uniaxial anisotropy is superimposed on it. With the decrease in temperature one can see that the ratio of the cubic and uniaxial components changes and that the change increases below $150 \mathrm{~K}$. In addition to
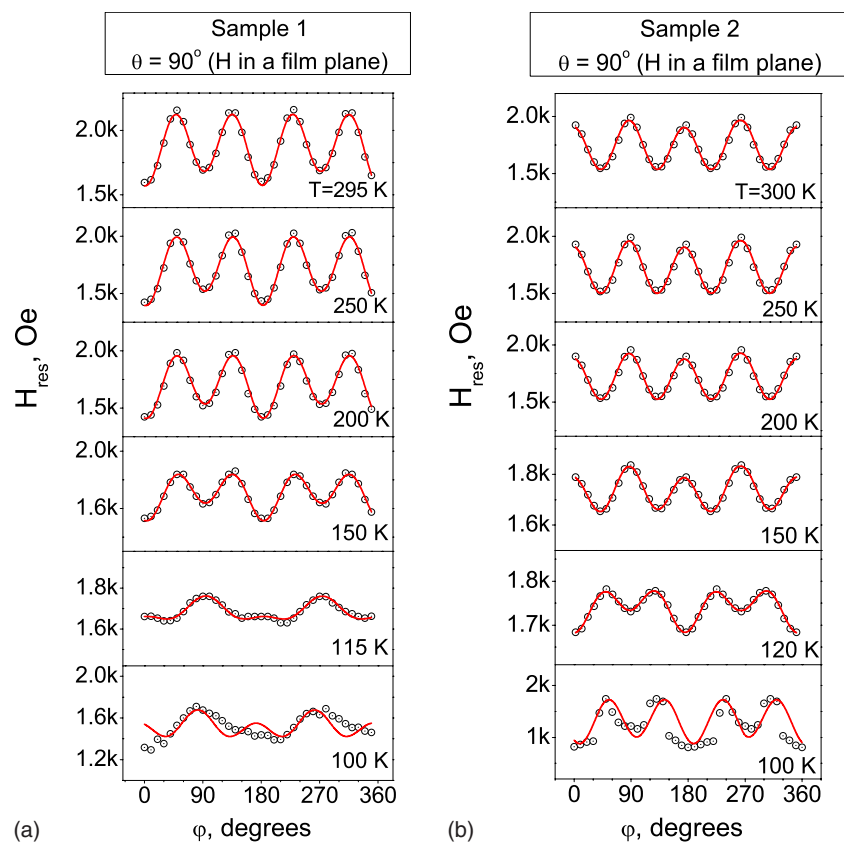

FIG. 2. (Color online) Angular dependence of the resonance field $H_{\mathrm{R}}$ for various temperatures for (a) sample 1 and (b) sample 2. Solid lines are the results of fitting according to formula (1).
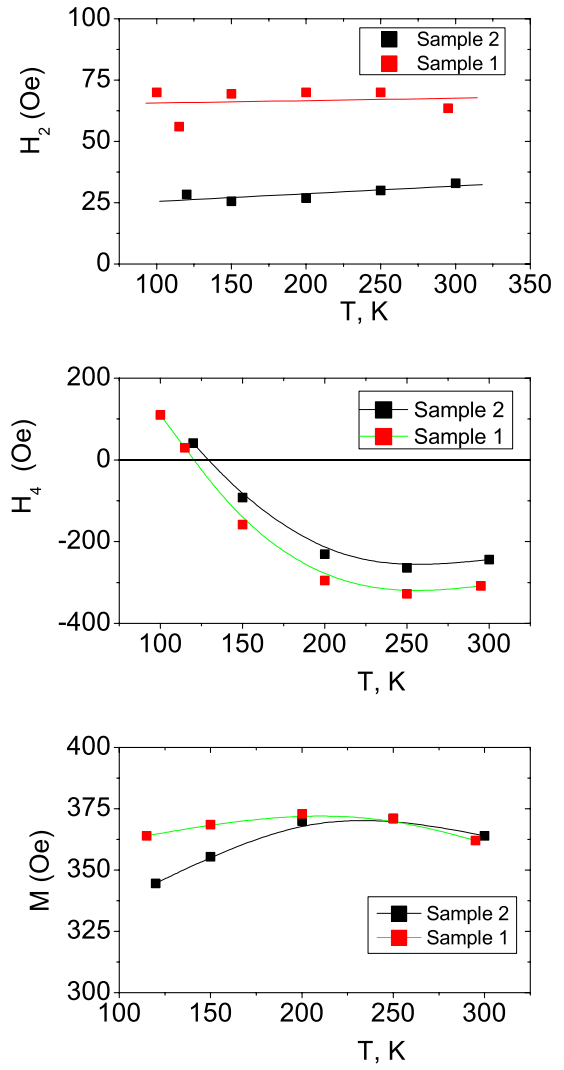

FIG. 3. (Color online) Temperature dependences of $H_{2}, H_{4}$ fields and $M$ as determined from the fitting procedure with the use of expression (1).

this, one can also see that sample 1 has a stronger uniaxial anisotropy component than sample 2. Apart from that, both samples display similar characteristics.

To process the experimental $H_{\mathrm{R}}$ versus $\varphi$ data, we consider the density of free energy as a sum of Zeeman, shape, cubic, and uniaxial-in-plane terms (similar to that reported in Ref. 14) and obtain a formula for the in-plane $(\theta=\pi / 2)$ resonance frequency,

$$
\begin{aligned}
(\omega / \gamma)^{2}= & \left\{H+H_{2} \cos (2 \varphi)+H_{4} \cos \left[4\left(\varphi+\alpha_{c}\right)\right]\right\}\{H \\
& +4 \pi M+H_{2} \cos ^{2} \varphi+0.25 H_{4}\left[\cos 4\left(\varphi+\alpha_{\mathrm{c}}\right)\right. \\
& +3]\} .
\end{aligned}
$$

Here, $\mathrm{H}_{4}$ and $\mathrm{H}_{2}$ are the fourth order cubic and second order uniaxial anisotropy fields, respectively, and $\alpha$ is the angle between the uniaxial easy axis and [010] axis (see Fig. 1). In the process of fitting, $\alpha_{\mathrm{c}}$ was set fixed $\left(\alpha_{\mathrm{c}}=\pi / 4\right.$ for sample 1 and 0 for sample 2). Results of the fitting are shown as solid lines in Fig. 2.

In Fig. 3, we compare the temperature variation in the uniaxial anisotropy field $\left(H_{2}\right)$ and cubic anisotropy field $\left(H_{4}\right)$ for both samples, as determined from the fitting procedure. $\mathrm{H}_{4}$ varies gradually with decreasing temperature for both samples and shows a crossover from negative to positive values at around $130 \mathrm{~K}$. These observations are in line with those for single crystalline magnetite. ${ }^{13,17}$ In bulk magnetite, it is well known that at $130 \mathrm{~K}, K_{4}$ changes sign. ${ }^{17}$ The magnitude of $\mathrm{H}_{2}$ at $300 \mathrm{~K}$ was found to be 63 and 32 Oe for sample 1 and sample 2, respectively. The $H_{2}$ for both samples shows little or no dependence on temperature, re- 

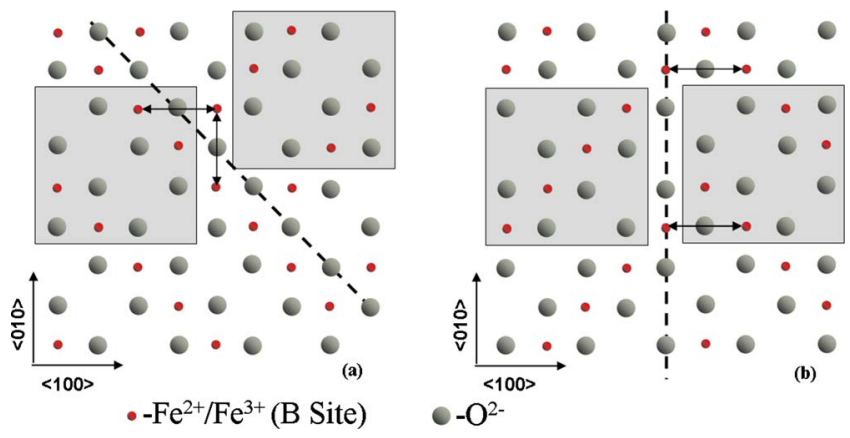

FIG. 4. (Color online) Schematic diagram of an APB formed in $\mathrm{Fe}_{3} \mathrm{O}_{4}$ films at the step edge on a vicinal $\mathrm{MgO}(100)$ substrate when the third B-layer on the lower terrace merges with the first $\mathrm{B}$-layer of $\mathrm{Fe}_{3} \mathrm{O}_{4}$ on upper terraces. Two cases are shown for the miscut direction of (a) $\langle 011\rangle$ and (b) $\langle 010\rangle$ crystallographic directions. The direction of step edge is marked with a dashed line. $\mathrm{Fe}-\mathrm{O}-\mathrm{Fe}$ interactions are marked with arrows in the figure for both kinds of APBs.

maining the same throughout the temperature range studied. Sample 1 has a stronger in-plane uniaxial anisotropy than sample 2 .

The perpendicular resonance field $\left(\theta=0^{\circ}\right)$ is about $8 \mathrm{kOe}$ for both the samples and its behavior is similar to that observed in Ref. 17 for a $47.5 \mathrm{~nm}$ thick magnetite film grown on single crystalline $\mathrm{MgO}$ substrate. This changes slightly with decreasing temperature until a certain value $\left(T_{\mathrm{v}}\right.$ $\sim 102 \mathrm{~K}$ ) is crossed. At this temperature, a sudden drop in the resonance field is observed, which is attributed to the Verwey transition. As in the case described in Ref. 17, the values of the $T_{\mathrm{v}}$ for the films are lower than the $T_{\mathrm{v}}$ for the bulk magnetite $\left(T_{\mathrm{v}}=123 \mathrm{~K}\right)$.

To explain the nature of the step-induced anisotropy in ultrathin magnetic films, Chuang et al. ${ }^{18}$ used the Néel pair anisotropy model. For the case of magnetite films, however, it has been shown that the use of this model alone is not enough to describe all the peculiar features observed. ${ }^{14}$ Furthermore, this model is not able to explain the strong effect of the miscut direction on the uniaxial component, as is reported above. Thus, additional mechanisms should be taken into account. At this stage, we should look more attentively at the effect of miscut direction on the geometry and magnetic structure of the APBs, which are induced by step edges. Earlier, Arora et al. ${ }^{11}$ measured the magnetoresistance parallel and perpendicular to the miscut direction and showed that the APBs would preferentially align to the step edges. As shown by Margulies et al. ${ }^{10}$ intrasublattice antiferromagnetic exchange dominates across the boundaries.

In Fig. 4, we show the schematic diagram of the formation of APBs when magnetite film is grown on $\mathrm{MgO}$ substrate that is miscut along $\langle 011\rangle$ or $\langle 010\rangle$ crystallographic directions. One can see that there are fewer $\mathrm{Fe}-\mathrm{O}-\mathrm{Fe}$ antiferromagnetic interactions in the APB formed on the $\mathrm{MgO}$ (100) substrate with a miscut direction of $\langle 010\rangle$ than that formed on a miscut along $\langle 011\rangle$. This suggests that exchange coupling across the APBs plays an important role in determining the strength of the uniaxial anisotropy component, and that it is this interaction which may be responsible for the effects observed in the samples under discussion.

\section{CONCLUSIONS}

We have investigated the role of miscut direction on the in-plane uniaxial magnetic anisotropy of epitaxial magnetite thin films. We find that the film grown on $\langle 011\rangle$ direction of miscut $\mathrm{MgO}$ possesses a stronger uniaxial anisotropy component than the one grown on $\langle 010\rangle$ miscut $\mathrm{MgO}$. The step induced uniaxial anisotropy is found to have very little temperature dependence. The different magnitudes of the uniaxial components observed for the films grown on the two miscut directions is related to the greater number of antiferromagnetic exchange interactions associated with the stepedge induced APB when it is formed on $\langle 011\rangle$ miscut $\mathrm{MgO}$ as compared to the $\langle 010\rangle$ miscut $\mathrm{MgO}$.

\section{ACKNOWLEDGMENTS}

The authors are grateful to the Science Foundation Ireland (SFI) for the financial support to carry out the work under Contract No. 00/PI.1/C042.

${ }^{1}$ J. Oster, M. Kallamayer, L. Wiehl, H. J. Elmers, and H. Adrian, J. Appl. Phys. 97, 014303 (2005).

${ }^{2}$ I. V. Shvets, H. C. Wu, V. Usov, F. Cuccureddu, S. K. Arora, and S. Murphy, Appl. Phys. Lett. 92, 023107 (2008).

${ }^{3}$ R. K. Kawakami, E. J. Escorcia-Aparicio, and Z. Q. Qiu, Phys. Rev. Lett. 77, 2570 (1996).

${ }^{4}$ T. Leeb, M. Brockmann, F. Bensch, S. Miethaner, and G. Bayreuther, J. Appl. Phys. 85, 4964 (1999).

${ }^{5}$ J. Chen and J. L. Erskine, Phys. Rev. Lett. 68, 1212 (1992).

${ }^{6}$ R. K. Kawakami, M. O. Bowen, H. J. Choi, E. J. Escorcia-Aparicio, and Z. Q. Qiu, Phys. Rev. B 58, R5924 (1998).

${ }^{7}$ V. Usov, S. Murphy, and I. V. Shvets, J. Appl. Phys. 95, 7312 (2004).

${ }^{8}$ B. B. Maranville, A. L. Shapiro, and F. Hellman, Appl. Phys. Lett. 81, 517 (2002).

${ }^{9}$ F. Walz, J. Phys.: Condens. Matter 14, R285 (2002).

${ }^{10}$ D. T. Margulies, F. T. Parker, M. L. Rudee, F. E. Spada, J. N. Chapman, P. R. Aitchison, and A. E. Berkowitz, Phys. Rev. Lett. 79, 5162 (1997).

${ }^{11}$ S. K. Arora, R. G. S. Sofin, and I. V. Shvets, Phys. Rev. B 72, 134404 (2005).

${ }^{12}$ T. Kasama, R. E. Dunin-Borkowski, and W. Eerenstein, Phys. Rev. B 73, 104432 (2006).

${ }^{13}$ S. Kale, S. M. Bhagat, S. E. Lofland, T. Scabarozi, S. B. Ogale, A. Orozco, S. R. Shinde, T. Venkatesan, B. Hannoyer, B. Mercey, and W. Prellier, Phys. Rev. B 64, 205413 (2001)

${ }^{14}$ L. McGuigan, R. C. Barklie, R. G. S. Sofin, S. K. Arora, and I. V. Shvets, Phys. Rev. B 77, 174424 (2008).

${ }^{15}$ R. G. S. Sofin, S. K. Arora, and I. V. Shvets, J. Magn. Magn. Mater. 316, e969 (2007).

${ }^{16}$ A. I. Tovstolytkin, A. M. Pogorily, Yu. I. Dzhezherya, V. V. Dzyublyuk, and D. J. Mapps, J. Phys.: Condens. Matter 21, 386003 (2009).

${ }^{17}$ P. A. A. van der Heijden, M. G. van Opstal, C. H. W. Swüste, P. H. J. Bloemen, J. M. Gaines, and W. J. M. de Jonge, J. Magn. Magn. Mater. 182, 71 (1998).

${ }^{18}$ D. S. Chuang, C. A. Ballentine, and R. C. O’Handley, Phys. Rev. B 49, 15084 (1994). 